\title{
Latitudinal and longitudinal dispersion of energetic auroral protons
}

\author{
D. A. Lorentzen \\ University Studies on Svalbard (UNIS), N-9170 Langyearbyen, Norway \\ E-mail: dag.lorentzen@unis.no
}

Received: 21 April 1999 / Revised: 2 July 1999 / Accepted: 20 July 1999

\begin{abstract}
Using a collision by collision model from Lorentzen et al., the latitudinal and longitudinal dispersion of single auroral protons are calculated. The proton energies varies from 1 to $50 \mathrm{keV}$, and are released into the atmosphere at $700 \mathrm{~km}$ altitude. The dipole magnetic field has a dip-angle of 8 degrees. Results show that the main dispersion region is at high altitudes $(300-350 \mathrm{~km})$ and occurs during the first few charge exchange collisions. As the proton travels further down the atmosphere the mean free path becomes smaller, and as a result the spreading effect will not be as pronounced. This means that the first few charge exchange collisions fully determines the width of both the latitudinal and longitudinal dispersion. The volume emission rate was calculated for energies between 1 and $50 \mathrm{keV}$, and it was found that dayside auroral hydrogen emissions rates were approximately 10 times weaker than nightside emission rates. Simulations were also performed to obtain the dependence of the particle dispersion as a function of initial pitch-angle. It was found that the dispersion varies greatly with initial pitch-angle, and the results are summarized in two tables; a main and an extreme dispersion region.
\end{abstract}

Key words. Ionosphere (auroral ionosphere; particle precipitation) - Space plasma physics (transport processes)

\section{Introduction}

Energetic protons penetrating the atmosphere will charge exchange with the surrounding atmospheric species. In this process the proton may pick up an electron and become a magnetically neutral hydrogen atom. This atom may travel vast distances across the magnetic fieldlines, before it collides with one of the atmospheric species where it may lose an electron and become a magnetically constrained proton. These two processes, the charge capture and charge stripping processes, are the main reason for the diffuse auroral features seen when observing the proton/hydrogen aurora.

Knowing the lateral dispersion of an incoming proton beam is essential when trying to solve the proton/hydrogen transport equations, though very little work has been done on this subject. The beam spreading effect is obtained by including a spreading factor in the one dimensional transport models (e.g., Basu et al., 1990; Shen, 1993). In a collision by collision model (e.g. Monte Carlo) the dispersion can be calculated since it is possible to keep track of the coordinates of each individual proton/hydrogen atom from the injection point down to the stop height.

The most referenced work in this context is the paper by Davidson (1965), where studies are made on the latitudinal spread of auroral protons. He finds that by using an isotropic injection pitch-angle distribution, precipitation of proton/hydrogen in the atmosphere over an area as wide as $600 \mathrm{~km}$ occurs. Injection energies of 5, 10 and $20 \mathrm{keV}$ were used. Davidson (1965) also states that he had to extrapolate the cross sections used from 5 to $20 \mathrm{keV}$, so that the results obtained may have rather considerable errors. We now have reliable cross sections for the range $100 \mathrm{eV}$ to $600 \mathrm{keV}$. In a research note Johnstone (1972), obtained an approximate expression for the spreading of a fine proton beam, based on the results of Davidson (1965). Since 1972 several models on proton transport have been made, but none of these models have included, or been able to include, any calculations on the dispersion of proton/hydrogen particles.

The most recent of these proton/hydrogen transport models, includes a one dimensional model (Jasperse and Basu, 1982), a numerical approach on coupled proton/ hydrogen transport equations (Basu et al., 1990), and an implicit finite differencing scheme (Shen, 1993). Basu et al. (1993) and Strickland et al. (1993) presented the first self consistent electron-proton-hydrogen transporttheoretical model, by solving the three coupled transport 
equations. The beam spreading effect is taken into account in these models by introducing a spreading factor. Kozelov (1993) using Monte Carlo calculations and Jasperse (1997) using a coupled set of two Boltzmann equations, both made calculations on the latitudinal beam spreading effect for a proton/hydrogen beam. Galand et al. (1997) presented a multistream approach to the two coupled Boltzmann transport equations, which included angular redistributions and magnetic mirroring effects.

In this work we will show that by using a collision by collision model, we are able to calculate both the latitudinal and longitudinal dispersion of single protons. In order to investigate the spreading we look at single monoenergetic, monodirectional protons with different initial energies and pitch-angle. The results will then be compared with the results of both Kozelov (1993) and Jasperse (1997).

\section{Short description of model}

We use the model described by Lorentzen et al. (1998) which is a collision by collision model, where single particles are injected into the atmosphere with a set of given initial values. These include pitch-angle, phase angle, energy and height. There are also some initial parameters that govern the atmosphere and surroundings. They include magnetic field inclination, observers view angle relative to the magnetic field and position of the injection point of the particles. The MSIS-86 thermospheric model (Hedin, 1987) is used as the model atmosphere, and the three major species $\mathrm{N}_{2}, \mathrm{O}_{2}$ and $\mathrm{O}$ makes up the atmosphere. The International Geomagnetic Reference Field (IGRF Revision, 1987) is used as a model magnetic field and considered dipolar, but with no curvature on the field lines themselves. The pitchangle for the proton particle is influenced by the magnetic field due to the conservation of the magnetic moment, and the pitch-angle for the hydrogen atom is influenced by the non-parallel field lines. As a result, most particles with pitch-angles above $65-70^{\circ}$ will be back scattered (Lorentzen et al., 1998). The effect of inelastic collisions on the pitch-angle is considered negligible (Lorentzen, 1993). Six different collision processes are used for the proton and hydrogen, including ionization and excitation for both $\mathrm{H}^{+}$and $\mathrm{H}$, and charge capture for $\mathrm{H}^{+}$and charge stripping for H. Eighteen different collision cross sections are used, and the collisions are determined using a weighted random selection.

A three dimensional coordinate system with $Y$ pointing towards geomagnetic south, $Z$ pointing to geographic zenith, and $X$ completing the right hand system, is used for this study. The magnetic field lines are placed in the $Y-Z$ plane (geomagnetic meridian plane), and have a constant angle of inclination $\beta$ tilted southward. This angle is often referred to as the dip angle, and is typically in the order of 10 degrees in the auroral zone. For a proton spiraling down the field lines the deviation in $x, y$ and $z$ coordinates are given by $x^{\prime}=x$

$y^{\prime}=y-\mathrm{d} s \cos \theta \sin \beta$

$z^{\prime}=z-\mathrm{d} s \cos \theta \cos \beta$,

where $\mathrm{d} s$ is the line segment traversed by the proton through the atmosphere, until a new collision occurs, $\theta$ is the pitch-angle of the proton, and $\beta$ the dip angle of the magnetic field lines. We have no motion in the $X$ direction and the minus sign in the $Y$ direction arises since the magnetic field lines are tilted southwards.

The deviation for the hydrogen atom, involves a slightly more complicated expression, because the hydrogen atom is magnetically neutral, and hence, not constrained by the magnetic field lines. This means that both the $X$ and $Y$ values might change, and that the deviation must depend on the azimuthal angle, or phase angle, as well as the pitch-angle and the inclination of the magnetic field lines;

$x^{\prime}=x+\mathrm{d} s \sin \theta \cos \phi \cdot(\cos \beta-\sin \beta)$

$y^{\prime}=y+\mathrm{d} s \sin \theta \sin \phi \cdot(\cos \beta+\sin \beta)$

$h^{\prime}=h+\mathrm{d} s \cos (\theta-\beta)$

where $\theta$ is the pitch-angle, $\phi$ the phase angle and $\beta$ is the dip angle. The phase angle was assumed to vary randomly throughout the atmosphere. The choice of a random phase angle was made after making simulations where a new phase angle was calculated from the speed and direction of the proton/hydrogen particle for each new collision. A power spectrum analysis of these calculated phase angles showed a power spectrum similar to that of a random group of elements.

\section{Proton/hydrogen dispersion}

Two sets of simulations were made. The first set (simulation set $A$ ) was made with monoenergetic protons with energies of $1,2,5,10,20$ and $50 \mathrm{keV}$, and with isotropic pitch-angle distributions, consistent with rocket and satellite observations (Søraas et al., 1974; Miller and Whalen, 1976; Urban, 1981) over the lower hemisphere. The second set of simulations (simulation set $B$ ) were made with monoenergetic protons with identical initial energies as simulation set $A$, but with static initial pitch-angles ranging from 1 to $90^{\circ}$ for each initial energy. The pitch-angles for each proton/hydrogen particle was allowed to vary according to the first adiabatic invariance (proton) and curvature of the magnetic field (hydrogen) as the particle penetrated the atmosphere.

In both these simulations the protons were injected at an altitude of $700 \mathrm{~km}$ with geographic coordinates 78.2N, 15.6E. (Longyearbyen, Svalbard.) Due to the dip angle of the magnetic field lines $\left(8^{\circ}\right.$, tilted southward) the particles injected at $78.2 \mathrm{~N}, 15.6 \mathrm{E}$ tends to travel poleward of the injection point, indicating that auroral hydrogen features seen in the zenith of an observation site in the Northern Hemisphere has an injection point equatorward of that site. 


\subsection{Simulation set $A$}

For simulation set $A$, the modeled data are presented as a scatter plot with latitude/longitude versus height. Each point in the plot indicates the collision position of a proton/hydrogen particle with one of the constituents of the atmosphere. A Gaussian density function is calculated for each scatter plot, and the contours of the Gaussian density are then plotted on top of the scatter plots. These contours are sometimes referred to as concentration ellipsoids since they indicate regions where the particle density is most concentrated. The concentration ellipsoids are found by calculating the covariance matrix of the elements in the scatter plot. The eigenvalues, $\lambda_{i}$, and the eigenvectors, $\boldsymbol{e}_{i}$, of the density matrix yields the sizes and rotation respectively, of the principal axes of the ellipse. On the order of $60 \%$ of the particles will be contained within the concentration ellipsoids.

Figures 1 and 2 gives the particle positions at the third and last collision for particles with energies from 1 to $50 \mathrm{keV}$, with isotropic pitch-angle distribution. For the third collision it is apparent that the collision altitude occurs around 300 to $350 \mathrm{~km}$, and that the collision altitude is approximately independent of energy. This is probably due to the low ionospheric particle density above these altitudes, i.e., the penetrating particles have a low probability for collisions regardless of initial energy at these altitudes.

It is also apparent that the particles are dispersed poleward of the injection point for the latitudinal deviation $(78.2 \mathrm{~N})$, and dispersed symmetrically around the longitudinal injection point (15.6E). The reason for the asymmetry in the latitudinal case is the southwardtilted magnetic field. The proton particles are magnetically constrained and will follow the magnetic field lines until a charge exchange occurs. Looking at the width of the concentration ellipsoids, we see that for the latitudinal case the width is almost constant with a value about $100 \mathrm{~km}$ for all injection energies. For the longitudinal case, the width seems to be constant with a value about $70 \mathrm{~km}$ for injection energies above $2 \mathrm{keV}$, but with smaller dispersion widths for $<2 \mathrm{keV}$ simulations.

For the last collision we see that the collision altitude varies dramatically with increasing energies, but with the same asymmetry and symmetry for latitudinal and longitudinal dispersion, respectively, as with the third collision. The width follows the same trend as with the third collision, i.e., an almost constant width, independent of energies for the latitudinal case, and with a more varying width in the longitudinal case. When comparing the widths for the third and last collisions at the same injection energies, we see that the last collision region has a dispersion width around 10 to $30 \%$ wider than where the 3 collision occurs. This is within the uncertainty of the concentration ellipsoids, so we can conclude that the dispersion width of a proton/hydrogen beam injected at $700 \mathrm{~km}$ is determined within the first few charge exchange collisions.

Figure 3 displays the mean penetration depth of injected protons with energies ranging from $0.1 \mathrm{keV}$ to $100 \mathrm{keV}$. The mean stop altitudes range from approx- imately $220 \mathrm{~km}$ for the $0.1 \mathrm{keV}$ particles down to $105 \mathrm{~km}$ for the $100 \mathrm{keV}$ particles. We see that the $2 \mathrm{keV}$ particles reach down to the region between $130 \mathrm{~km}$ to $150 \mathrm{~km}$ where the atmospheric density starts its exponential growth. There is an almost linear dependence on stop altitude versus energy before and after that region. From this region and below we find the main emission regions for hydrogen aurora.

\subsection{Differential volume emission rate}

The differential volume emission rate as a function of height can be found by

$D_{n m}=\frac{A_{n m}}{\sum_{m=1}^{n-1} A_{n m}} N_{\mathrm{M}}(h) \sigma_{\mathrm{eff}}^{n m}(v) n(h, \theta, \phi) F(r, v)$,

in units of (photons $\mathrm{cm}^{-3} \mathrm{~s}^{-1} \mathrm{sr}^{-1} \mathrm{keV}^{-1}$ ), when assuming negligible collisional quenching. $A_{n m}$ is the spontaneous transition probability between the levels $n$ and $m$, $N_{\mathrm{M}}$ atmospheric number density, $\sigma_{\text {eff }}^{n m}$ the effective excitation cross section, $n(h, \theta, \phi)$ the pitch-angle distribution and $F(r, v)$ the directional particle flux. In Fig. 4, the volume emission rate versus altitude for initial energies between 1 and $50 \mathrm{keV}$ is shown. There are strong diurnal variations in the precipitating energy of auroral protons, and using Doppler shifted emission/ wavelength profiles the 'bulk' energy can be deduced by looking at the emission intensity and half width at full maximum (HWFM) of the line profiles. Nightside auroral particles are more energetic than dayside auroral particles, and typical values for dayside proton precipitation are in the order of 1 to $3 \mathrm{keV}$, while nightside precipitations are in the range 5 to $10 \mathrm{keV}$. (see Sigernes et al., 1993; 1996; Sigernes, 1996; Lorentzen et al., 1998). From Fig. 4 we can deduce that typical dayside emission altitudes are from 130 to $160 \mathrm{~km}$, while nightside emission altitudes range from 110 to $130 \mathrm{~km}$. We also see that the dayside volume emission rate is on the order of 10 times weaker than the nightside emission rate for equal flux magnitudes.

\subsection{Modeled auroral hydrogen arc}

Figures 5 and 6 show an auroral hydrogen arc with the volume emission rate as a function of altitude and proton dispersion for particles with 'bulk' energy of $5 \mathrm{keV}$ and isotrophic pitch-angle. In Fig. 5 the geomagnetic east-west (E-W) direction is plotted, and we note that the emissions are symmetric with respect to the ' 0 ' injection point and that the main emission region is located within a $140-150 \mathrm{~km}$ region. Figure 6 shows the geomagnetic north-south (N-S) direction and we note the asymmetric emission distribution with respect to the ' 0 ' injection point, which is due to the tilt angle of the magnetic field lines. The $\mathrm{N}-\mathrm{S}$ emission region covers a $200 \mathrm{~km}$ wide area. The modeled auroral hydrogen arc shows that a typical altitude distribution for the given initial conditions is 30 to $40 \mathrm{~km}$. 

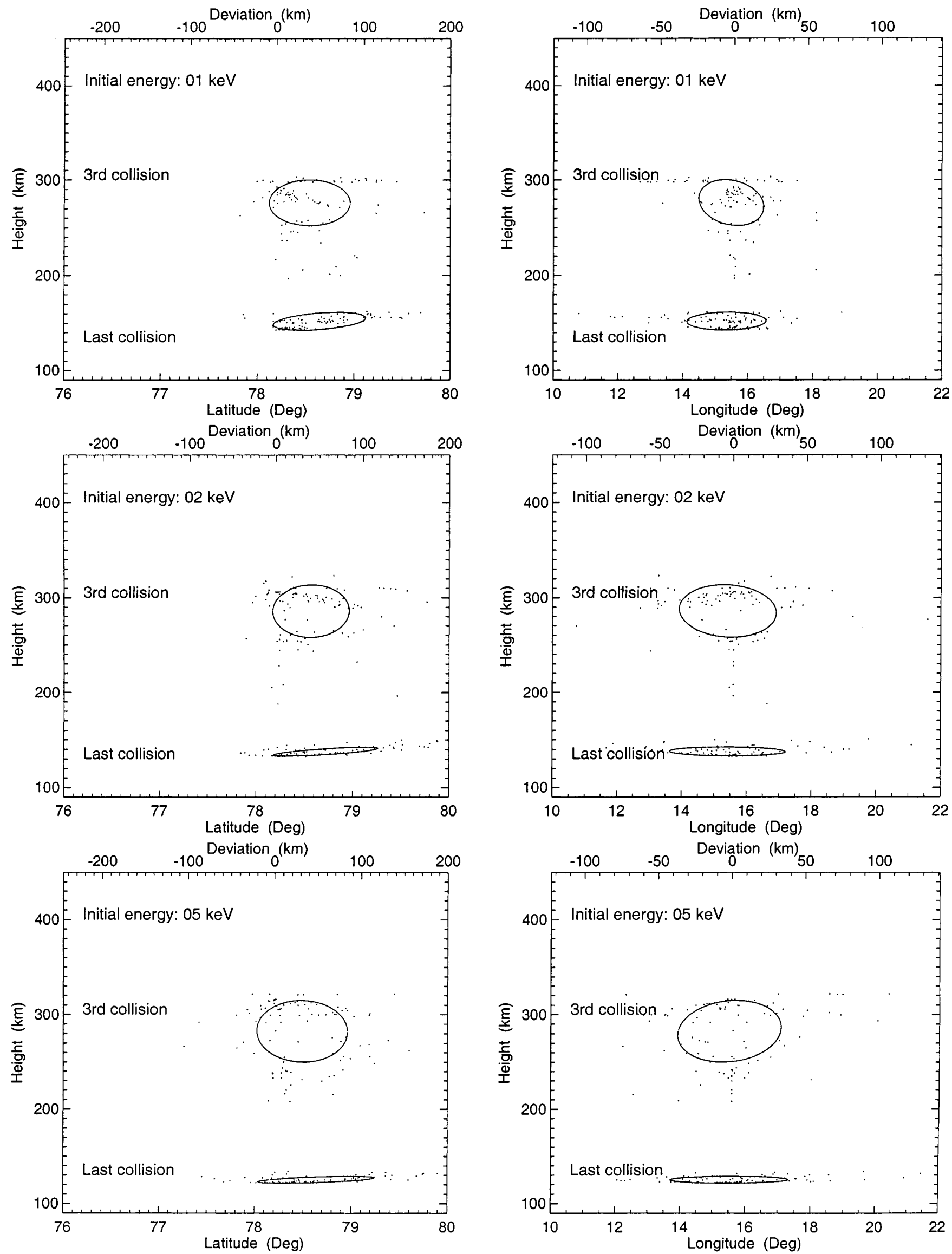

Fig. 1. Latitudinal and longitudinal dispersion at third and last collision, for initial energies of 1, 2 and $5 \mathrm{keV}$ 

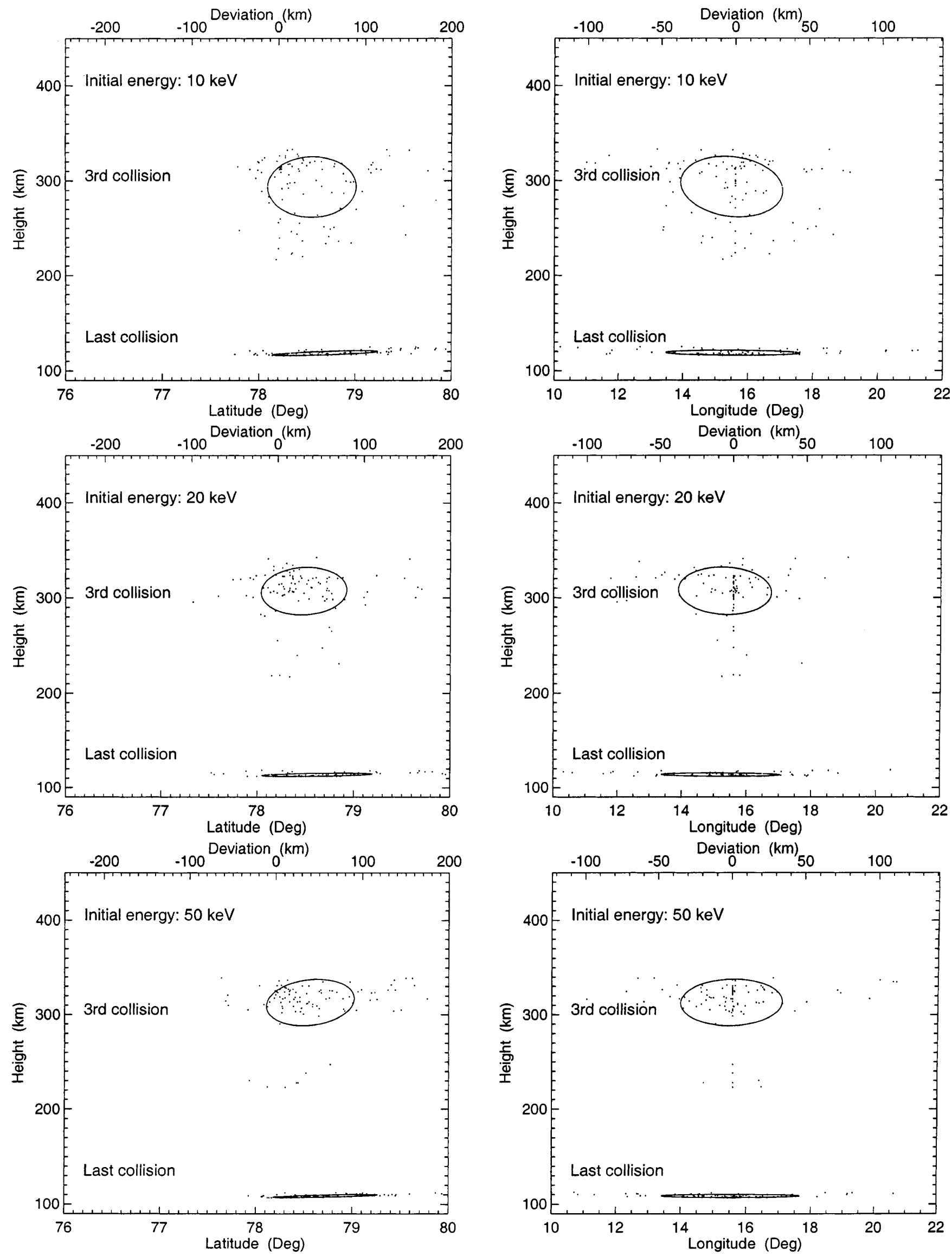

Fig. 2. Latitudinal and longitudinal dispersion at third and last collision, for initial energies of 10, 20 and $50 \mathrm{keV}$ 


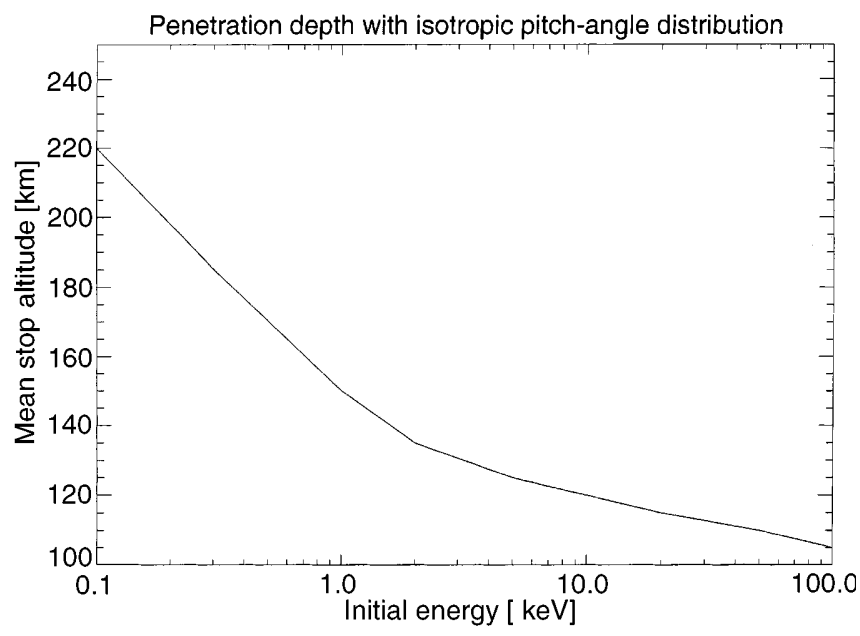

Fig. 3. Penetration depth of auroral proton/hydrogen particles

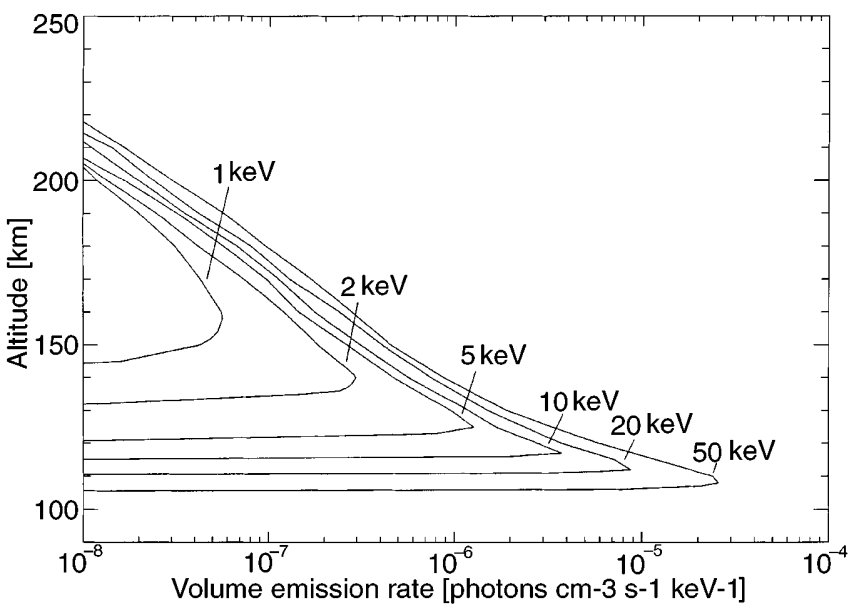

Fig. 4. Volume emission rate versus altitude, for protons with 1, 2, 5, 10,20 and $50 \mathrm{keV}$ initial energy
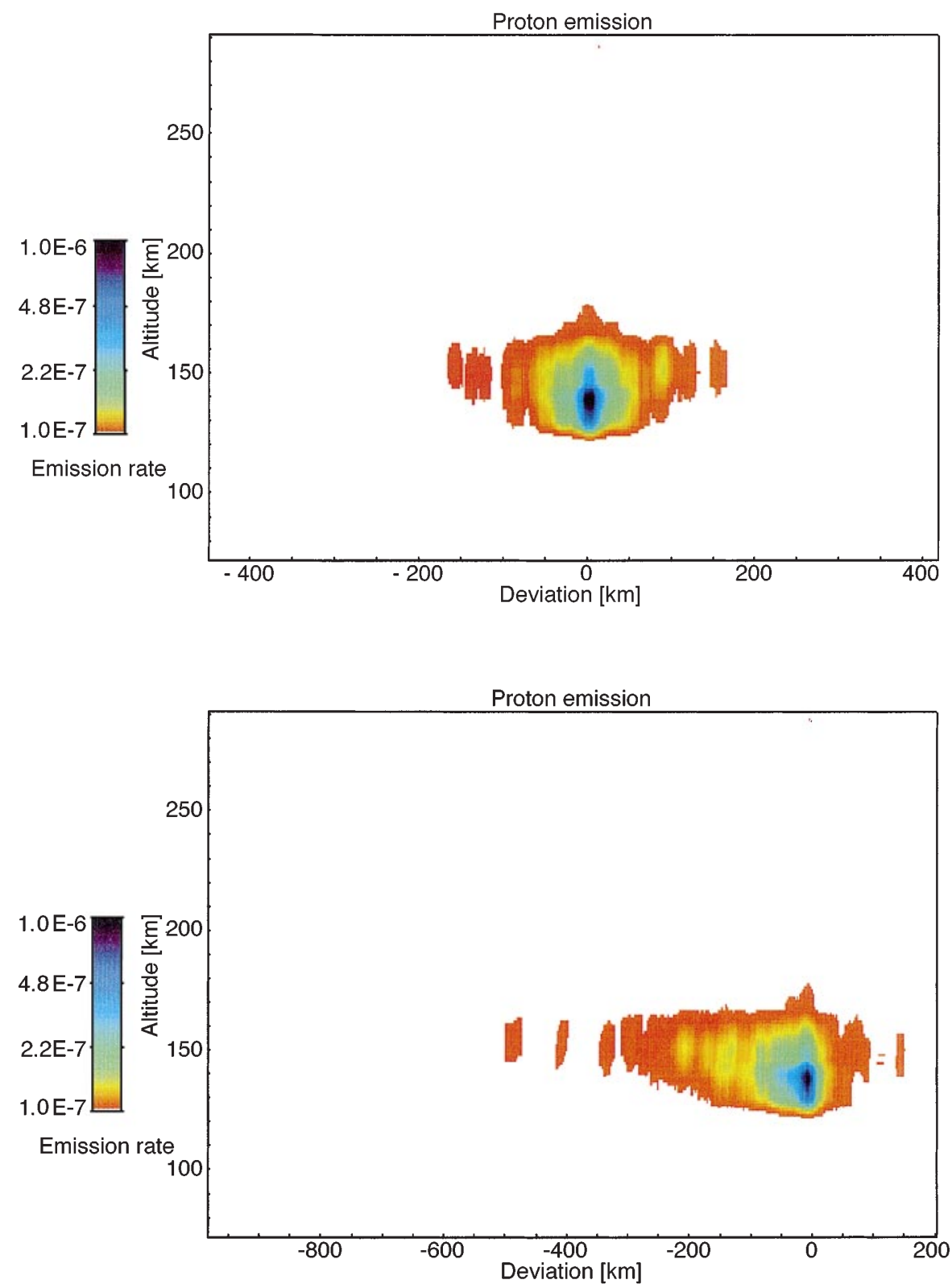

Fig. 5. Modeled auroral hydrogen arc in the east-west geomagnetic direction, for particles with injection energies of $5 \mathrm{keV}$ and isotropic pitch angle distribution
Fig. 6. Modeled auroral hydrogen arc in the north-south geomagnetic direction, for particles with injection energies of $5 \mathrm{keV}$ and isotropic pitch angle distribution 


\subsection{Simulation set $B$}

The purpose of simulation set $B$ is to investigate the dispersion of the proton/hydrogen particles as a function of initial pitch-angle. The simulation was conducted for the same range of energies as simulation set $A$, and each of the initial energies ran with static pitch-angles of $1,5,10,20,30,40,50,60,70$ and $80^{\circ}$. It was found that the dispersion as a function of initial pitch-angle was approximately energy independent, so the results could be broken in to two parts an extreme dispersion region and a main dispersion region. More than $90 \%$ of the particles fall within the first region, while around $60 \%$ of the particles fall within the latter.

Table 1 gives the modeled values for the extreme dispersion region. The first feature we would like to point out is that the latitudinal dispersion is greater than the longitudinal dispersion. The reason for this is the tilt of the magnetic field in the geomagnetic meridian plane. The magnetically constrained protons follows the tilted field lines in this plane, but this effect is absent in the longitudinal case. We also note that for both the latitudinal and longitudinal case there is a pronounced jump in dispersion width above $60^{\circ}$ initial pitch-angle. A likely reason for this is that the particles with pitchangles above $60^{\circ}$ are allowed to travel vast distances in a low density atmospheric region, so that collisions occur more infrequently than for particles with lower initial pitch-angles. The latter particles reach denser regions faster, which results in smaller mean free paths for these particles.

Table 2 gives the modeled values for the main dispersion region. This is the region where most of the particles producing emissions are located. Note that the particles with initial pitch-angles above $70^{\circ}$ most likely will not produce any light measurable by ground-based optical instruments (Lorentzen et al., 1998). This is because these particles will most likely mirror before they reach emission altitudes. Using Table 2, we are then able to deduce that the light producing particles from a beam of protons emanating from one single injection point are likely to be contained within a $200(\mathrm{~N}-\mathrm{S})$ by $140(\mathrm{E}-\mathrm{W}) \mathrm{km}$ region for particles with maximum initial pitch-angles around 60 to $70^{\circ}$. Note that this is only a

Table 1. Extreme dispersion region

\begin{tabular}{lcc}
\hline $\begin{array}{l}\text { Initial } \\
\text { pitch-angle }\left(^{\circ}\right)\end{array}$ & $\begin{array}{l}\text { Longitudinal } \\
(\mathrm{E}-\mathrm{W})(\mathrm{km})\end{array}$ & $\begin{array}{l}\text { Latitudinal } \\
(\mathrm{N}-\mathrm{S})(\mathrm{km})\end{array}$ \\
\hline 1 & $2( \pm 0.5)$ & $3( \pm 1)$ \\
5 & $10( \pm 2.5)$ & $15( \pm 4)$ \\
10 & $25( \pm 6)$ & $40( \pm 10)$ \\
20 & $50( \pm 12)$ & $75( \pm 19)$ \\
30 & $80( \pm 20)$ & $120( \pm 30)$ \\
40 & $115( \pm 29)$ & $180( \pm 45)$ \\
50 & $155( \pm 38)$ & $245( \pm 61)$ \\
60 & $210( \pm 52)$ & $325( \pm 81)$ \\
70 & $400( \pm 100)$ & $600( \pm 150)$ \\
80 & $800( \pm 200)$ & $1000( \pm 250)$
\end{tabular}

E-W is geomagnetic east-west direction, N-S is geomagnetic northsouth direction
Table 2. Main dispersion region

\begin{tabular}{lcc}
\hline $\begin{array}{l}\text { Initial } \\
\text { pitch-angle }\left({ }^{\circ}\right)\end{array}$ & $\begin{array}{l}\text { Longitudinal } \\
(\mathrm{E}-\mathrm{W})(\mathrm{km})\end{array}$ & $\begin{array}{l}\text { Latitudinal } \\
(\mathrm{N}-\mathrm{S})(\mathrm{km})\end{array}$ \\
\hline 1 & $1( \pm 0.3)$ & $2( \pm 0.5)$ \\
5 & $5( \pm 1.3)$ & $8( \pm 2)$ \\
10 & $10( \pm 2.5)$ & $15( \pm 4)$ \\
20 & $20( \pm 5)$ & $35( \pm 9)$ \\
30 & $40( \pm 10)$ & $70( \pm 18)$ \\
40 & $60( \pm 15)$ & $105( \pm 26)$ \\
50 & $80( \pm 20)$ & $140( \pm 35)$ \\
60 & $105( \pm 26)$ & $180( \pm 45)$ \\
70 & $155( \pm 38)$ & $220( \pm 55)$ \\
80 & $200( \pm 50)$ & $300( \pm 75)$ \\
\hline
\end{tabular}

E-W is geomagnetic east-west direction, N-S is geomagnetic northsouth direction

rough estimate, and may vary considerably e.g., according to the pitch-angle distribution of the beam.

\subsection{Model comparison}

Although there are different initial conditions and parameters, it is interesting to compare the latitudinal beam spreading results of the present work with those obtained by Kozelov (1993) with his Monte-Carlo calculations and Jasperse (1997) with his solutions of the two coupled Boltzmann equations.

Taking Jasperse's (1997) Fig. 2 as a starting point, he calculated the normalized beam half widths for a proton and hydrogen beam in the altitude range $600-280 \mathrm{~km}$, for particles with initial energies of $8 \mathrm{keV}$. In the lower part of this region, Jasperse's (1997) full beam width at the $1 / \mathrm{e}$ point can be estimated to be on the order of $80 \mathrm{~km}$. Using Fig. 9 in Kozelov (1993), where a proton beam with initial energy of $10 \mathrm{keV}$ is used, the full beam width of $80 \%$ of the particles at $280 \mathrm{~km}$ altitude is on the order of $140 \mathrm{~km}$. Using Fig. 2 in the present work, we see that the altitude region around $280 \mathrm{~km}$ corresponds nicely to the third collision region for a proton beam with $10 \mathrm{keV}$ initial energy. The full beam width of the concentration ellipsoid (i.e., approximately $60 \%$ of the particles) is on the order of $100 \mathrm{~km}$ in this case.

For models that are so different in method and composition, there is a good correlation between the results of the different papers. The Kozelov (1993) model and the model used here are more similar than the model presented in the Jasperse (1997) paper, and, not surprisingly, they have a better agreement in the model results. The Jasperse (1997) paper seem to have slightly underestimated the total beam width as compared with the other two, but this can probably be attributed to different model setups, such as model atmosphere, magnetic field and other initial parameters.

Note that Jasperse (1997) also states that very little beam spreading occurs from 280 to $150 \mathrm{~km}$ for a given energy distribution, which agrees well with the results of this study. There are however disagreements with the Kozelov (1993) paper, which states that there is an energy dependence of the beam width below $280 \mathrm{~km}$ for 
particles with energies between 10 and $50 \mathrm{keV}$. There are several important factors that have to be taken into consideration in this problem, and they are all connected; a collision, at any given altitude, is determined by the initial pitch-angle and initial energy of the particle. Knowing this, the probability for a certain collision to happen can be found from the collisional cross sections (which is a function of the particle energy) and the atmospheric density (which is a function of the altitude). The most important collisional cross sections for the beam spreading problem are charge capture and charge stripping. When looking at the cross sections it is important to be aware of which target gas we are looking at. Kozelov (1993) uses a model atmosphere consisting of $\mathrm{N}_{2}$, while the present model uses atomic and molecular oxygen in addition. This is an important point since in the $10-50 \mathrm{keV}$ energy range there is factor of 5 difference in the charge stripping cross section for $\mathrm{N}_{2}$, while in the same energy range there is only a factor of 2 difference between the two extremities for $\mathrm{O}$ and $\mathrm{O}_{2}$. Hence the use of an $\mathrm{N}_{2}$-only atmosphere will favor beam spreading for low energy particles more than a three species atmosphere would do. For particle energies above $50 \mathrm{keV}$ one should see a noticeable difference since the charge capture cross section decreases substantially above this energy for all gases. Hence, it is the authors view that there is not a large energy dependence in the beam width for particles in the energy range $10-50 \mathrm{keV}$.

\section{Conclusion}

Using the model presented in Lorentzen et al. (1998), we have investigated the latitudinal and longitudinal dispersion of auroral protons:

1. Particle positions were found using the pitch-angle, phase angle and the inclination of the magnetic field lines.

2. Simulation set $A$ was performed using monoenergetic particles with energies of 1, 2, 5, 10,20 and $50 \mathrm{keV}$, with isotropic pitch-angle distribution. The results were presented as scatter plots with concentration ellipsoids superimposed. These ellipsoids indicates the highest density region of the dispersed particles. Particles dispersed along the N-S geomagnetic meridian tends to travel poleward of the injection point. The dispersion seems to be determined during the first few charge exchange collisions, and the dispersion seems to be independent of energy.

3 . The penetration depth (mean stop altitude) was calculated for particles with initial energies from 0.1 to $100 \mathrm{keV}$. The mean stop altitude ranged from $220 \mathrm{~km}$ down to $105 \mathrm{~km}$ for the given energies. The mean stop altitude was also found to coincide with the main emission region.

4. The main emission region was found to be located between 130 to $160 \mathrm{~km}$ altitude for dayside auroral hydrogen emissions, while the nightside auroral hydrogen emissions where found to be located in the region between 110 and $130 \mathrm{~km}$. The dayside emission rate is generally 10 times weaker than the nightside emission rate for equal flux magnitudes.

5. Simulation set $B$ was performed using monoenergetic particles with the same energies as set $A$, but with static initial pitch-angles ranging from 1 to $80^{\circ}$. The results of this simulation were presented in two tables, showing the extreme and main dispersion regions as a function of initial pitch-angle. We found that the particle dispersion is greatly affected by the initial pitch-angle, and that the latitudinal dispersion is greater than the longitudinal due to the tilt of the magnetic field in the geomagnetic meridian plane. Particles with initial pitchangles above 60 to $70^{\circ}$ have a very wide dispersion width, but are most likely to mirror before they enter the main emission altitudes, and hence will not produce any light.

6. A typical dispersion region for a beam of auroral protons injected at the same injection point was found to be contained within a $200 \mathrm{~km}(\mathrm{~N}-\mathrm{S})$ by $140 \mathrm{~km}(\mathrm{E}-\mathrm{W})$ region.

7. A fairly good agreement on the latitudinal beam spread with two other models were also obtained, which substantiate the validity of the present model.

Acknowledgements. This work was funded in parts by NASA grant NAG5-693 to the Geophysical Institute, University of Alaska, Fairbanks, and The University Courses on Svalbard. The author would like to thank Dr. C. Deehr, Geophysical Institute, University of Alaska, Fairbanks for his help with evaluating this manuscript.

The Editor-in-chief thanks M. H. Rees and another referee for their help in evaluating this paper.

\section{References}

Basu, B., J. R. Jasperse, and N. J. Grossbard, A Numerical solution of the coupled proton-H atom transport equations for the proton aurora, J. Geophys. Res., 95, 19 069, 1990.

Basu, B., J. R. Jasperse, D. J. Strickland, and R. E. Daniell, Jr., Transport-theoretic model for the electron-proton hydrogen atom aurora, 1. Theory, J. Geophys. Res., 98, 21 517, 1993.

Davidson, G. T., Expected spatial distribution of low-energy protons precipitated in the auroral zones, J. Geophys. Res., 70, 1061, 1965.

Galand, M., J. Lilensten, and W. Kofman, Proton transport model in the ionosphere 1. Multistream approach of the transport equations, J. Geophys. Res., 102, 22 261, 1997.

Hedin, A. E., MSIS-86 thermospheric model, J. Geophys. Res., 92, 4649, 1987.

IGRF Revision 1987, J. Geomagn. Geoelectr., 39, 773, 1987.

Jasperse, J. R., Transport theoretic solutions for the beamspreading effect in the proton-hydrogen aurora, Geophys. Res. Lett., 24, 1415, 1997.

Jasperse, J. R., and B. Basu, Transport theoretic solutions for auroral proton and $\mathrm{H}$ atom fluxes and related quantities., J. Geophys. Res., 87, 811, 1982.

Johnstone, A. D., The spreading of a proton beam by the atmosphere, Planet. Space Sci., 20, 292, 1972.

Kozelov, B. V., Influence of the dipolar magnetic field on transport of proton-H atom fluxes in the atmosphere, Ann. Geophysicae, 11, 697, 1993.

Lorentzen, D. A., A three dimensional model and ground-based measurements of $\mathrm{H}_{\alpha}$ and $\mathrm{H}_{\beta}$ Doppler hydrogen line profiles. PhD. Thesis, University of Tromsø, Norway, 1993.

Lorentzen, D. A., F. Sigernes, and C. S. Deehr, Modelling and observations of dayside auroral hydrogen emission Doppler profiles, J. Geophys. Res., 17 479, 1998. 
Miller, J. R., and B. A. Whalen, Characteristics of auroral precipitation observed from sounding rockets, J. Geophys. Res., 81, 147, 1976.

Shen, D., Proton transport and auroral optical emissions. PhD Thesis, Geophysical Institute, University of Alaska, Fairbanks, 1993.

Sigernes, F., Estimation of initial auroral proton energy fluxes from Doppler profiles, J. Atmos. Terr. Phys., 58, 1871, 1996.

Sigernes, F., D. A. Lorentzen, C. S. Deehr, and K. Henriksen, Modulation of the auroral proton spectrum in the upper atmosphere, J. Atmos. Terr. Phys., 55, 1289, 1993.

Sigernes, F., G. Fasel, C. S. Deehr, R. W. Smith, D. A. Lorentzen, L.T. Wetjen and K. Henriksen, Calculations and ground-based observations of pulsed proton events in the dayside aurora, J. Atmos. Terr. Phys., 58, 1281, 1996.

Strickland, D. J., R. E. Daniell, Jr., J. R. Jasperse, and B. Basu, Transport-theoretic model for the electron-proton-hydrogen atom aurora, 2. Model results, J. Geophys. Res., 98, 21 533, 1993.

Søraas, F., H. R. Lindalen, K. Måseide, A. Egeland, T. A. Sten, and D. S. Evans, Proton precipitation and the $\mathrm{H}_{\beta}$ emission in a postbreakup auroral glow, J. Geophys. Res., 79, 1851, 1974.

Urban, A., Measurements of low energy auroral ions, Planet. Space Sci., 29, 1353, 1981 